\title{
A programmable pAgo nuclease with RNA target preference from the psychrotolerant bacteria Mucilaginibacter paludis
}

\author{
Wenqiang $\mathrm{Li}^{1, \dagger}$, Yang $\mathrm{Liu}^{1, \dagger}$, Fei Wang ${ }^{1, *}$ and Lixin $\mathrm{Ma}^{1, *}$ \\ 1 State Key Laboratory of Biocatalysis and Enzyme Engineering, Hubei Collaborative Innovation \\ Center for Green Transformation of Bio-resources, Hubei Key Laboratory of Industrial Biotechnology, \\ School of Life Sciences, Hubei University, Wuhan, Hubei 430062, China \\ * To whom correspondence should be addressed. Tel: +86-27-50865628; Fax: +86-27-88666349; \\ Email: malixing@hubu.edu.cn
}

Correspondence may also be addressed to Fei Wang. Email: wangfei@hubu.edu.cn

The authors wish it to be known that, in their opinion, the first two authors should be regarded as joint First Authors

\begin{abstract}
Argonaute (Ago) proteins are programmable nuclease found in both eukaryotes and prokaryotes. Prokaryotic Argonaute proteins (pAgos) share a high degree of structural homology with eukaryotic Argonaute proteins (eAgos) and eAgos are considered to evolve from pAgos. However, the majority of studied pAgos prefer to cleave DNA targets, and eAgos exclusively cleave RNA targets. Here, we characterize a novel pAgo, MbpAgo, from psychrotolerant bacteria Mucilaginibacter paludis that can be programmed with DNA guides and prefers to cleave RNA targets rather than DNA targets. MbpAgo can be active at a wide range of temperatures $\left(4-65^{\circ} \mathrm{C}\right)$. In comparison with previously studied pAgos, MbpAgo is able to utilize 16-nt long 5'phosphorylated and 5'hydroxylated DNA guides for efficient and precise cleavage and displays no obvious preference for the 5'end nucleotide of a guide. Furthermore, the cleavage efficiency can be regulated by mismatches in the central and 3'supplementary regions of the guide. MbpAgo can efficiently cleave highly-structured RNA targets using both 5'phosphorylated and 5'hydroxylated DNA guides in the presence of $\mathrm{Mg}^{2+}$ or $\mathrm{Mn}^{2+}$. In conclusion, we have demonstrated that MbpAgo is a unique programmable nuclease that has a strong preference for RNA targets, with great potential applications in the field of nucleic acid biotechnology.
\end{abstract}




\section{INTRODUCTION}

Argonaute proteins (Agos) are present in all three domains of life, and they use small DNA or RNA guides for recognition and sometimes cleavage of complementary nucleic acid targets (1). As the key components of RNA interference (RNAi) pathways, eukaryotic Argonautes (eAgos) participate in posttranscriptional gene regulation and antivirus defence (2). Similarly to eAgos, prokaryotic Argonautes (pAgos) have been shown to protect cells from mobile genetic elements, including plasmids and phages (3-6). In addition, a recent study showed that pAgos may function in the DNA replication (7), which suggests that the cellular functions of pAgos are diverse and remains to be further explored.

Genomic studies showed that pAgos are more diverse than eAgos (8), and pAgos can be classified into long pAgos, short pAgos and PIWI-RE (9). Despite these divergent cellular functions and diversity, pAgos and eAgos adopt a highly conserved six domains architecture, including the $\mathrm{N}$ terminal (N), linker 1 (L1), middle (MID), linker 2 (L2), PIWI-Argonaute-Zwille (PAZ), and P elementinduced wimpy testis (PIWI) domains (5). Molecular structures of Agos showed that the 5' end and the 3 ' end of nucleic acid guides are anchored in the MID and PAZ domains, respectively $(10,11)$. Agos with endonuclease activity have a catalytic tetrad DEDX ( $X$ is $\mathrm{D}, \mathrm{H}, \mathrm{N}$, or $\mathrm{K}$ ) in the PIWI domain, which is essential for binding divalent metal ions and responsible for catalysis $(5,12,13)$. After binding with small nucleic acid guides, active Agos can precisely cleave the complementary targets between the 10 ' and 11' nucleotides of the guide nucleic acid (14).

While eAgos exclusively cleave RNA targets, previously characterized pAgos prefer to cleave DNA targets. Initially, pAgos were characterized from thermophilic prokaryotes, including TtAgo (Thermus thermophilus) (3), PfAgo (Pyrococcus furiosus) (4), MpAgo (Marinitoga piezophile) (13), and MjAgo (Methanocaldococcus jannaschii) $(15,16)$, which can be programmed with DNA guides or RNA guides to cleave DNA targets or RNA targets effectively in elevated temperatures but not in moderate temperatures. Recently, active pAgos from mesophilic prokaryotes including CbAgo (Clostridium butyricum) (17,18), LrAgo (Limnothrix rosea) (17), SeAgo (Synechococcus elongatus) (19), KmAgo (Kurthia massiliensis) (20,21), CpAgo (Clostridium perfringens) and IbAgo (Intestinibacter bartlettii) (22) were characterized to search for a pAgo that can cleave double-stranded DNA (dsDNA) at moderate temperatures and be used for genome editing. Besides, some pAgos perform guideindependent target DNA processing activity, including TtAgo, MjAgo, CbAgo, LrAgo, and SeAgo $(15,17,19,23)$. Among them, KmAgo can utilize both DNA and RNA guides to cleave almost all types of nucleic acids at moderate temperatures, including single-stranded DNA (ssDNA), dsDNA, unstructured RNA and highly-structured RNA, but can only use 5'phosphorylated DNA (5'P-DNA) guide to cleave RNA with high efficiency (20).

The programmable binding and cleavage activities of pAgos are similar to that of the CRISPR-Cas system, which means pAgos can potentially be used in genome editing applications (9), molecular cloning (24), and nucleic acid detection (25-28). Though eAgos prefer to use small RNA guides to cleave RNA targets, there are only a few pAgos that have been reported to recognize RNA targets, and no pAgos with the ability to preferentially cleave RNA targets with high accuracies at mesophilic temperatures have been described to date. The search for pAgos capable of cleave RNA targets with high specificity and high efficiency at $37^{\circ} \mathrm{C}$, which could have widespread applications in RNA fields, 
is therefore of great importance. In this work, we characterize a novel pAgo, MbpAgo from Mucilaginibacter paludis, which is distantly related to other characterized pAgos, and contains the canonical catalytic tetrad in the PIWI domain (residues D566, E601, D635, and D768). We show that different from other pAgos, MbpAgo binds DNA guides to cleave RNA with high efficiency but DNA with very low efficiency at physiological temperatures. Furthermore, we demonstrated that MbpAgo can utilize both 5' phosphorylated and 5' hydroxylated DNA guides to cleave unstructured RNA and highly-structured RNA, which suggests that it can expand the toolkit for RNA-manipulation and detection.

\section{MATERIAL AND METHODS}

\section{Protein expression and purification}

The MbpAgo gene (WP_008504757.1; Mucilaginibacter paludis) and MbpAgo double mutant (MpbAgo_DM) (D566A, D635A) gene were synthesized by Wuhan Genecreate Biotechnology Co., Ltd and cloned into pET28a expression vectors. The MbpAgo and MbpAgo_DM proteins were expressed in E. coli Rosetta (DE3) (Novagen). Cultures were grown at $37^{\circ} \mathrm{C}$ in Luria-Bertani (LB) medium containing $50 \mu \mathrm{g} / \mathrm{ml}$ kanamycin and until $\mathrm{OD}_{600}$ reached 0.8 . MbpAgo expression was induced by the addition of isopropyl- $\beta$-D-1-thiogalactopyranoside (IPTG) to a final concentration of 0.5 $\mathrm{mM}$. Cells were incubated at $18^{\circ} \mathrm{C}$ for $16 \mathrm{~h}$ with continuous shaking for expression. Centrifugally collected cells were stored at a $-80^{\circ} \mathrm{C}$ refrigerator for further protein purification.

The cell pellet was resuspended in Buffer A (20 mM Tris- $\mathrm{HCl} \mathrm{pH} \mathrm{7.4,} 500$ mM NaCl, 20 mM imidazole) supplemented with $1 \mathrm{mM}$ of phenylmethylsulfonyl fluoride (PMSF) and disrupted by sonication (SCIENTZ-IID. $400 \mathrm{~W}, 2 \mathrm{~s}$ on/4 s off for $15 \mathrm{~min}$ ). The lysate was clarified by centrifugation and the supernatant was loaded onto Ni-NTA agarose resin for $50 \mathrm{~min}$ with rotation. The beads were washed with Buffer $A$, then with the same buffer containing $50 \mathrm{mM}$ imidazole and eluted with Buffer $A$ containing $150 \mathrm{mM}$ imidazole. Fractions containing MbpAgo were concentrated by ultrafiltration using an Amicon 50K filter unit (Millipore) and purified on a Superdex 200 16/600 column (GE Healthcare) equilibrated with Buffer B (20 mM Tris- $\mathrm{HCl}$ pH 7.4, $500 \mathrm{mM} \mathrm{NaCl})$. Fractions containing MbpAgo were concentrated Amicon 50K filter unit (Merck Millipore), placed in Buffer B (20 mM Tris-HCl pH 7.4, 500 $\mathrm{mM} \mathrm{NaCl}$ ), aliquoted and flash-frozen in liquid nitrogen.

\section{Single-stranded nucleic acid cleavage assays}

The cleavage assays were performed using the synthetic guide and target (see Supplementary Table S1 for oligonucleotide sequences). 5'-FAM-labeled targets and 5'-P-labeled guides were synthesized for some experiments. The cleavage reactions were performed in PCR tubes at $37^{\circ} \mathrm{C}$ in a buffer containing $10 \mathrm{mM}$ HEPES- $\mathrm{NaOH}$ pH 7.5, $100 \mathrm{mM} \mathrm{NaCl}, 5 \mathrm{mM} \mathrm{MnCl}_{2}$, and $5 \%$ glycerol. To analyze the effect of various divalent cations, $5 \mathrm{mM} \mathrm{Mg}^{2+}, \mathrm{Ni}^{2+}, \mathrm{Co}^{2+}, \mathrm{Cu}^{2+}, \mathrm{Fe}^{2+}, \mathrm{Ca}^{2+}$, or $\mathrm{Zn}^{2+}$ were added instead of $\mathrm{Mn}^{2+} .800 \mathrm{nM}$ of MbpAgo was mixed with $400 \mathrm{nM}$ DNA guide or RNA guide and incubated for $10 \mathrm{~min}$ at $37^{\circ} \mathrm{C}$ for guide loading. Target nucleic acids were then added to the final concentration of $200 \mathrm{nM}$. For analysis of temperature dependence of RNA cleavage, MbpAgo was loaded with DNA 
guide for 10 min at $37^{\circ} \mathrm{C}$, the samples were transferred to indicated temperatures in a PCR thermocycler (T100, Bio-Rad), RNA target was added and the samples were incubated for $15 \mathrm{~min}$. All reactions were carried out at $37^{\circ} \mathrm{C}$ if not indicated. The reactions were stopped after indicated time intervals by mixing the samples with equal volumes $2 \times$ RNA loading dye (95\% Formamide, $18 \mathrm{mM}$ EDTA, and $0.025 \%$ SDS, and $0.025 \%$ Bromophenol Blue) and heated for $5 \mathrm{~min}$ at $95^{\circ} \mathrm{C}$. The cleavage products were resolved by $20 \%$ denaturing PAGE, stained with SYBR Gold (Invitrogen), visualized with Gel Doc ${ }^{\mathrm{TM}} \mathrm{XR}+$ (Bio-Rad), and analyzed by the Image J and Prism 8 (GraphPad) software. For reactions containing FAM labels, the gels were first visualized with Gel Doc ${ }^{\mathrm{TM}} \mathrm{XR}+(\mathrm{Bio}-$ Rad) and then stained with SYBR Gold.

\section{Highly-structured RNA cleavage assays}

The SARS-CoV-2 RdRp partial RNA (see Supplementary Table S2) were in vitro transcribed using T7 RNA polymerase (Thermo Fisher Scientific) and synthetic DNA templates carrying a T7 promoter sequence. The transcripts were treated with DNase I, gel-purified, and ethanol precipitated. DNA guides used for cleavage (Supplementary Table S3) were 5'-phosphorylated using T4 PNK (New England Biolabs) except when cleavage with 5'-OH guides. $1 \mu \mathrm{M}$ of MbpAgo was mixed with $1 \mu \mathrm{M}$ guide DNA and incubated for $10 \mathrm{~min}$ at $37^{\circ} \mathrm{C}$ for guide loading. Target RNA was then added to the final concentration of $250 \mathrm{nM}$ and incubated for $30 \mathrm{~min}$ at $37^{\circ} \mathrm{C}$ for cleavage. Reactions were stopped with $2 \times$ RNA loading dye and heated for $5 \mathrm{~min}$ at $95^{\circ} \mathrm{C}$. The cleavage products were resolved by $8 \%$ denaturing PAGE and stained with SYBR Gold.

\section{Electrophoretic mobility shift assay (EMSA)}

To examine the loading of guide onto MbpAgo, MbpAgo and 3'end FAM-labeled guide were incubated in $10 \mu \mathrm{l}$ of reaction buffer ( $10 \mathrm{mM}$ HEPES-NaOH pH 7.5, $100 \mathrm{mM} \mathrm{NaCl}, 5 \mathrm{mM} \mathrm{Mn}^{2+}$ ) for 30 $\min$ at $37^{\circ} \mathrm{C}$. The concentration of the guide was fixed as $100 \mathrm{nM}$, whereas the concentration of MbpAgo varied. Then the samples were mixed with $1 \mu \mathrm{l} 10 \times$ loading buffer $(250 \mathrm{mM}$ Tris-HCl $(\mathrm{pH} 7.5)$, $40 \%$ glycerol) and resolved by $10 \%$ native PAGE with $0.5 \times$ Tris-Borate-EDTA (TBE) buffer. Nucleic acids were visualized using Gel Doc ${ }^{\mathrm{TM}} \mathrm{XR}+$. To analyze the loading of the guide:target duplex, reactions contained MbpAgo_DM, 100 nM 3'end FAM-labeled guide, $100 \mathrm{nM}$ target, were combined for $10 \mu \mathrm{l}$ and incubated for $30 \mathrm{~min}$ at $37^{\circ} \mathrm{C}$. The concentration of MbpAgo_DM varied. Then the samples were mixed with $1 \mu \mathrm{l} 10 \times$ loading buffer and resolved by $10 \%$ native PAGE with $0.5 \times$ TBE. Nucleic acids were visualized using Gel Doc ${ }^{\mathrm{TM}} \mathrm{XR}+$. To determine the apparent dissociation constants $(\mathrm{Kd})$ for guide binding and guide:target duplex binding, the obtained gel images were analyzed with the NIH program ImageJ and Prism 8 (GraphPad) software. The data were fitted with the Hill equation with a Hill coefficient of 2-2.5. All the nucleic acids used in this study are listed in Supplementary Table S1.

\section{Co-purification nucleic acids}

Isolation of co-purification nucleic acids was carried out as described in ref. (18) with minor modifications. Briefly, $\mathrm{CaCl}_{2}$ and proteinase $\mathrm{K}$ (Zomanbio) were added to final concentrations of $5 \mathrm{mM}$ 
and $0.5 \mathrm{mg} / \mathrm{ml}$ to $2 \mathrm{mg}$ of purified MbpAgo in Buffer B. The sample was incubated for $50 \mathrm{~min}$ at $55^{\circ} \mathrm{C}$. The nucleic acids were separated from the organic fraction by adding Roti-phenol/chloroform/isoamyl alcohol pH 7.5-8.0 in a 1:1 ratio. The top layer was isolated and nucleic acids were precipitated using ethanol precipitation by adding $99 \%$ ethanol in a $1: 2$ ratio supplied with $0.5 \%$ linear polymerized acrylamide as a carrier. This mixture was incubated for $16 \mathrm{~h}$ at $-20^{\circ} \mathrm{C}$ and centrifuged in a table centrifuge at 13,000 rpm for $30 \mathrm{~min}$. Next, the nucleic acid pellet was washed with $500 \mu \mathrm{l}$ of $70 \%$ ethanol and solved in $50 \mu \mathrm{l}$ nuclease-free water. The purified nucleic acids were treated with either $100 \mu \mathrm{g} / \mathrm{ml}$ RNase A (Thermo Fisher Scientific), 2 units DNase I (NEB), or both for $1 \mathrm{~h}$ at $37^{\circ} \mathrm{C}$ and resolved on a denaturing urea polyacrylamide gel $(20 \%)$ and stained with SYBR Gold.

\section{RESULTS}

\section{MbpAgo prefers to cleave RNA rather than DNA with small DNA guides at ambient temperature}

MbpAgo (accession number WP_008504757.1 in the NCBI protein database) is distantly related to other characterized eAgos and pAgos (sequence identity < 20\%) (Figure 1A and S1D) and contains the canonical catalytic tetrad in the PIWI domain (residues D566, E601, D635, and D768) (Supplementary Figure S1D). To study the biochemical properties and in vivo functions of MbpAgo, we expressed and purified MbpAgo. Codon-optimized gene encoding MbpAgo was chemically synthesized and cloned into pET28a plasmid (Supplementary Figure S1A). In addition to the wild-type protein, we obtained its catalytically inactive variant (MbpAgo_DM) with substitutions of two out of four catalytic tetrad residues (D566A/D635A) (Supplementary Figure S1D). The protein was expressed in E. coli and purified using Ni-NTA-affinity and size-exclusion (see Supplementary Figure S1 and Materials and Methods for details). Examination of purified MbpAgo showed high purity of the samples (Supplementary Figure S1).

We first studied the nucleic acid specificity of MbpAgo in an in vitro cleavage assay using synthetic fluorescently labeled oligonucleotides targets (Figure 1B). MbpAgo was loaded with $18 \mathrm{nt}$ DNA or RNA guides containing a $5^{\prime}$ phosphate or $5^{\prime}$ hydroxyl group at $37^{\circ} \mathrm{C}$ for $10 \mathrm{~min}$ followed by the addition of complementary 5'end FAM-labeled 45-nt long single-stranded DNA or RNA targets (Figure 1B, Supplementary Table S1). After incubation for $30 \mathrm{~min}$ at $37^{\circ} \mathrm{C}$, the cleavage products were resolved by $20 \%$ denaturing gel (Figure 1C and 1D). MbpAgo uses both 5'phosphorylated DNA guide ( $5^{\prime} \mathrm{P}$ gDNA) and 5'hydroxylated DNA guide (5'OH-gDNA) to cleave RNA target (tRNA) (Figure 1C). Unexpectedly, no DNA target (tDNA) cleavage was observed (Figure 1D), and only very weak tDNA cleavage was observed even after incubation for $6 \mathrm{~h}$ (Supplementary Figure S1E). With the RNA guide, we did not observe any MbpAgo-mediated cleavage, neither of DNA nor RNA targets, even after incubation for $12 \mathrm{~h}$ (Figure 1C, 1D and Supplementary Figure S1E). This DNA-guided RNA target preference has not been observed in other eAgos or pAgos homologs, for eAgos prefer to use RNA guides to target RNA and pAgos prefer to use DNA guides to target DNA. Cleavage of the target strand by MbpAgo occurs after the 10th nucleotide counting from the 5'end of the 5'P-gDNA, consistent with previously characterized Ago homologs (Supplementary Figure S1F and S1G) (14). When guided with 5'OH-gDNA, target cleavage not only occurred between target position 10'-11' relative to the guide 5'end but also occurred at 1-2 nucleotides downstream of the canonical cleavage 
site (Supplementary Figure S1F and S1G), which is similar to that catalyzed by CbAgo (17) and $K m A g o ~(20)$. No cleavage activity was detected for a catalytically dead MbpAgo variant with substitutions of two of the tetrad residues (D566A/D635A) (Figure 1C). In summary, in contrast to previously characterized pAgos that prefer to target DNA, MbpAgo prefers to target RNA rather than DNA and can potentially be exploited for RNA manipulation.

\section{MbpAgo uses 5'OH-gDNA and 5'P-gDNA to cleave RNA with almost the same efficiency and is active over a wide range of temperatures}

The majority of pAgos strongly prefer 5'-phosphorylated nucleic acid guides $(5,17,20)$. As shown in Figure $1 \mathrm{C}$ and Supplementary Figure S2A, MbpAgo can use 5'P-gDNA and 5'OH-gDNA to cleave almost all RNA target in $30 \mathrm{~min}$, but with different precision. We next tested RNA target cleavage kinetics and the results showed that MbpAgo uses 5'OH-gDNA and 5'P-gDNA to cleave RNA with almost the same efficiency (Figure 2A). To explore the temperatures range at which MbpAgo is active, we tested the temperature-dependent of RNA cleavage activity. MbpAgo bound with 5'P-gDNA had comparable levels of RNA cleavage activity between 25 and $65^{\circ} \mathrm{C}$, and retained good activity to cleave RNA at $4-20^{\circ} \mathrm{C}$. For $5^{\prime} \mathrm{OH}-g D N A$, the activity was enhanced with increasing temperature from 4 to $50^{\circ} \mathrm{C}$, and then decreased rapidly with higher temperature (Figure $2 \mathrm{~B}$, Supplementary Figure S2B and $\mathrm{S} 2 \mathrm{C}$ ), suggesting that interactions with the 5'-phosphate may be essential to stabilize the binary MbpAgo-guide complex at elevated temperatures (17).

\section{Divalent cations and guide lengths affect the cleavage of MbpAgo}

We next tested which divalent cations MbpAgo can utilize to mediate DNA-guided RNA target cleavage. MbpAgo can utilize $\mathrm{Mn}^{2+}, \mathrm{Mg}^{2+}, \mathrm{Ni}^{2+}$ and $\mathrm{Co}^{2+}$ as cation, with $\mathrm{Mn}^{2+}$ being a better cation than other cations (Figure $2 \mathrm{C}$ and $2 \mathrm{~F}$ ). Titration of $\mathrm{Mn}^{2+}$ ions showed that MbpAgo was active between 0.01 and $10 \mathrm{mM} \mathrm{Mn}^{2+}$ (Figure 2D and 2G), regardless of whether the guide is 5'P-gDNA or $5^{\prime} \mathrm{OH}-g D N A$. Titration of $\mathrm{Mg}^{2+}$ ions showed that MbpAgo was active between 0.1 and $10 \mathrm{mM} \mathrm{Mg}^{2+}$ (Figure $2 \mathrm{E}$ and $2 \mathrm{H}$ ), regardless of whether the guide is $5^{\prime} \mathrm{P}-\mathrm{gDNA}$ or $5^{\prime} \mathrm{OH}-\mathrm{gDNA}$.

We further investigated MbpAgo cleavage efficiency using DNA guides of different lengths, revealing that MbpAgo was most active with 15-20 nt DNA guides, with a lower efficiency observed with longer or shorter guides (Figure 3A). Similar to that of KmAgo, the cleavage position was shifted if shorter (11-13 nt) and longer (21-40) 5'P-gDNAs were used (20,21). Interestingly, the cleavage only occurred between the 10th and 11th guide positions when using 15-17 nt long 5'OH-gDNA, while the cleavage position was shifted if shorter (10-14 nt) or longer (18-30 nt) 5'OH-gDNA were used (Figure $3 A)$. In conclusion, the length of the guide has no great influence on the RNA cleavage activity but has an influence on the cleavage precision.

To test whether MbpAgo is a single-turnover enzyme or a multiple-turnover enzyme, we monitored the cleavage assays in a time course using variable [MbpAgo-gDNA]: RNA target ratios (Figure 3D) at $37^{\circ} \mathrm{C}$ and $50^{\circ} \mathrm{C}$, respectively. We observed a rapid burst of activity during the first minute, but this stage was followed by a slow steady-state and finally only a fraction of the target was cleaved at $37^{\circ} \mathrm{C}$ during the 60 min course of the reaction (Figure 3D, left panel; Supplementary Figure S2F). In 
contrast, when the reaction was performed at $50^{\circ} \mathrm{C}$ almost all RNA was cleaved during 60 min, indicative of multiple-turnover reaction (Figure 3D, right panel; Supplementary Figure S2G). In conclusion, while MbpAgo function as a multiple-turnover enzyme, its steady-state rate may be limited by product release and exchange of target molecules like that of CbAgo and KmAgo $(18,21)$.

\section{Effects of 5' guide nucleotide and guide-target mismatches on target cleavage}

Previous studies demonstrated that the first nucleotide in the guide strand is bound in the MID pocket of Ago, and many studied eAgos and pAgos have a certain preference for it (5). To determine if MbpAgo has specificity for the first nucleotide of the guide, we also tested four guide variants with different first nucleotides but otherwise identical sequences. Similar to that of CbAgo and KmAgo, we observed no changes in the cleavage efficiency and rate when MbpAgo was loaded with 5'phosphorylated DNA guides with different first nucleotides (Figure $3 \mathrm{C}$ and Supplementary Figure S2E) $(17,21)$.

Mismatches between the guide and target have effects on the cleavage activities of Ago proteins $(17,20,29)$. To determine the sequence specificity of MbpAgo, we analyzed the effects of mismatches between the guide and target strands on its RNA cleavage activity. We designed a set of DNA guides, each containing a single-nucleotide or dinucleotide mismatches at a certain position (Supplementary Table S1), and tested them in the RNA cleavage reaction with MbpAgo (Figure 3B and Supplementary Figure S2D). Despite mismatch at position 9 decreased the cleavage efficiency, other single-nucleotide mismatches had no significant effect on cleavage. However, dinucleotide mismatches at position 10-15 dramatically reduced target cleavage, which suggested that MbpAgo can potentially be programmed for specific cleavage of desired target sequence.

\section{Binding analysis of guides and targets by MbpAgo}

Our results indicated that MbpAgo could use 5'OH DNA guides to cleave the target RNA with similar efficiency in comparison to 5'P DNA guides in figure 2A. Previously structural and bioinformatic studies showed that the MID and PAZ domain anchors the 5'end of a guide by a conserved sixamino-acid motif, and the first two residues of this motif might be important for the function of different groups of pAgos (8). Multiple sequence alignment of the 5'end guide binding pocket of the MID domain from MbpAgo with several other characterized Ago proteins showed that the first two residues of most characterized Ago proteins are YK, while the first two residues of MbpAgo are HK (Supplementary Figure S3A). With the structure of CbAgo with its 5'P DNA guide and DNA target (PDB ID: 6qzk, identity 18.8\%) as a template, we used the SWISS-MODEL portal to build the structure of MbpAgo, which displays four typical domains of pAgo protein structure (Figure 4A). The $3 \mathrm{D}$ model aliged to CbAgo revealed that first two amino acid residues in the specific motif involved in interaction with the guide 5 '-end, which consistent with the multi-sequence alignment results (Figure $4 \mathrm{~A}$ and Supplementary Figure S3A). The imidazole of the H482 could stack on the first base at the guide 5'-end in the MID domain of MbpAgo like the Y472 in the CbAgo. For 5'OH DNA guide, the presence of potential contacts between the MID Domain and the guide 5 '-end might compensate for the loss of interactions with the 5'-phosphate in MbpAgo (17). 
We further measured the dissociation constants $(\mathrm{Kd})$ for guide binding by MbpAgo using EMSA (Figure 4B and Supplementary Figure S3B). MbpAgo has similar Kd values for 5'P DNA guides 253.7 $\mathrm{nM}(95 \% \mathrm{Cl}: 214.3$ to $277.0 \mathrm{nM})$ and 5'OH DNA guides $291.8 \mathrm{nM}$ (95\% Cl: 245.9 to $317.0 \mathrm{nM}$ ), which may be the reason why MbpAgo is able to use both 5'P and 5'OH DNA guides for efficiently RNA target cleavage. We also measured the dissociation constants for guide: target duplex binding by MbpAgo using EMSA (Figure 4C and Supplementary Figure S3C). As shown in Figure 4C and Supplementary Figure S3C, MbpAgo is able to bind both 5'P-gDNA: tRNA duplex and 5'P-gDNA: tDNA duplex with comparable affinity, which suggested that the weak DNA target cleavage activity may be not due to target binding affinity. Furthermore, we analyzed the nucleic acids that co-purify with MbpAgo after expression in E. coli (Supplementary Figure S1H). Small DNA was not observed in RNase A-treated samples, while RNAs of undefined length were observed in DNase I-treated samples. They are suspected to be non-specifically bound nucleic acids, as has previously been described for purification of TtAgo and PfAgo $(3,4)$.

\section{MbpAgo can use 5'P-gDNA and 5'OH-gDNA to cleave highly structured SARS-CoV-2 RdRp RNA}

The coronavirus disease 2019 (COVID-19) pandemic, caused by the severe acute respiratory syndrome coronavirus 2 (SARS-CoV-2) virus, has highlighted the need for antiviral approaches. The SARS-CoV-2 virus belongs to a family of positive-sense RNA viruses, and Abbott et al reported a CRISPR-Cas13-based strategy for viral inhibition that can effectively degrade RNA from SARS-CoV-2 sequences (30). To explore whether MbpAgo can cleave RNA efficiently, we designed a set of gDNAs corresponding to different regions of the highly conserved RNA-dependent RNA polymerase (RdRP) gene, which maintains the proliferation of SARS-CoV-2. We hypothesized that secondary structure of the SARS-CoV-2 RdRp RNA, as predicted by the NUPACK algorithm (Figure 5A) (31). MbpAgo was loaded with gDNAs corresponding to different regions of SARS-CoV-2 RdRp RNA that either maintain single-stranded conformation or are involved in extensive base-pairing (Figure 5A and 5B). Cleavage products were detected with all 5'P-gDNAs and $5^{\prime} \mathrm{OH}$-gDNAs when reactions were performed with 5 $\mathrm{mM} \mathrm{Mn}^{2+}$ (Figure 5C and 5D). Moreover, MbpAgo can cleave SARS-CoV-2 RdRp RNA at the expected position for all sites with $5 \mathrm{mM} \mathrm{Mg}^{2+}$, albeit to a little less efficient (Supplementary Figure S4A and S4B).

\section{DISCUSSION}

Most characterized mesophilic pAgos proteins were shown to strongly prefer DNA targets (17-20), and NgAgo (Natronobacterium gregory) was reported to only degrade RNA targets by an unknown mechanism with poor accuracy (32). In this study, we have characterized a novel prokaryotic Argonaute protein from the psychrotolerant bacteria Mucilaginibacter paludis (33). Though MbpAgo prefers to use the DNA guides like most characterized pAgos, MbpAgo has an unusually preference for cleaving RNA targets with high precision and efficiency at moderate temperatures but has very weak activity in cleaving DNA targets. We have shown that MbpAgo can target RNA under a wide range of reaction conditions. The efficiency and accuracy of cleavage are modulated by temperature, 
divalent ions, the phosphorylation and length of the DNA guides and its complementarity to the RNA targets.

In contrast to other studied Agos, MbpAgo is able to use $5^{\prime} \mathrm{OH}$ guides for RNA targets cleavage with almost the same efficiency as 5'P guides. Except for several pAgos, including MpAgo, exclusively bind $5^{\prime} \mathrm{OH}$ guides (13), the majority of eAgos and pAgos were shown to use 5'P guides for RNA cleavage, and multiple interacts between the 5'P group and the MID domain are observed in the structure of some Ago-guide complexes $(5,18)$. A bioinformatic study revealed several subtypes of the MID domain with substitutions of key residues involved in interactions with the 5 'end of guide molecule (8). The MID domain of most Agos, including CbAgo, RsAgo, and KmAgo, contains YK subtype, while MbpAgo contains HK subtype (Supplementary Figure S3A). As far as we know, MbpAgo might be the first studied pAgo with HK subtype in the motif. Furthermore, homology-based structural modeling suggests that interactions of the guide 5'end with the MID pocket are overall very similar for CbAgo and MbpAgo (Figure 4A). Therefore, interactions with other parts of the guide may be important for stabilize the 5'OH guide in MbpAgo. Moreover, MbpAgo binds 5'P ( 253.7 nM) and 5 'OH ( 291.8 nM) DNA guides with comparable binding affinities. The affinities are much lower in comparison with CbAgo ( $6.2 \mathrm{pM})$, MjAgo ( $\sim 3 \mathrm{nM})$, and RsAgo ( 42.6 $\mathrm{nM})$ but similar to hAgo2 ( 565 nM) (17,34-36). Furthermore, both DNA targets and RNA targets can be bound to MbpAgo with similar binding affinities. The conformational changes of MbpAgo in the process of binding to the DNA target may not make the nucleic acid duplex and the catalytic cleft close enough, resulting in the weak DNA cleavage activity $(29,37)$. Thus, structural studies will be necessary to determine the structural basis of the apparent preference for RNA targets. We were unable to co-purify DNA guides from MbpAgo heterologously expressed in E. coli. Possibly MbpAgo is not able to generate enough guides for its poor DNA cleavage activity or requires host factors for guide generation and/or loading (4). Future studies should focus on guides associated with MbpAgo expressed in Mucilaginibacter paludis.

MbpAgo is able to cleave RNA targets at a wide range of temperatures and still has good RNA cleavage activity at $4-20^{\circ} \mathrm{C}$, which may be due to MbpAgo is from a psychrotolerant bacteria. This suggests pAgos with good activity at mesophilic temperatures could be also found from psychrotolerant and psychrophilic bacterias. MbpAgo preferentially uses 15-18 nt long DNA guides and is mostly active as RNA endonuclease in the presence of $\mathrm{Mg}^{2+}$ or $\mathrm{Mn}^{2+}$, similarly to previously studied pAgos. Interestingly, we have shown that the efficiency and the position of cleavage can be modulated by the length of the guide. While the cleavage site is shifted if shorter or longer $5{ }^{\prime} \mathrm{OH}$ guides are used, the cleavage position is not shifted if $15-17 \mathrm{nt}$ long $5^{\prime} \mathrm{OH}$ guides are used. That means we can use MbpAgo to cleave RNA targets with high efficiency and precision by choosing $5^{\prime} \mathrm{OH}-g D N A$ of appropriate length. Moreover, the cleavage site is also shifted if $18 \mathrm{nt} 5^{\prime} \mathrm{OH}$ guides are used in some pAgos, including CbAgo, CpAgo, IbAgo, LrAgo and $\mathrm{KmAgo}$. Choosing $5^{\prime} \mathrm{OH}$ guides of appropriate length could use these pAgos to cleave targets with high efficiency and precision.

MbpAgo has no obvious preference for the 5'end nucleotide of a guide, which is similar to several other pAgos, including KmAgo and CbAgo $(17,20)$. Importantly, the cleavage activity of MbpAgo is greatly affected when introducing mismatches with the RNA target. We have shown that the cleavage efficiency can be regulated by mismatches in the central and 3'supplementary regions of the guide. 
Finally, we have demonstrated that MbpAgo can efficiently cleave highly-structured RNA targets using both 5'P-gDNAs and 5'OH-gDNAs in the presence of $\mathrm{Mg}^{2+}$ or $\mathrm{Mn}^{2+}$. Previously, eAgo from the budding yeast Kluyveromyces polysporus and pAgo from the mesophilic bacteria Kurthia masiliensis were used for highly structured RNA cleavage $(21,38)$. Different from their findings, the cleavage efficiency of $M b p A g o$ does not greatly depend on the secondary structure at $37^{\circ} \mathrm{C}$. Therefore, MbpAgo can be used for cleavage of complex RNA targets independently of the secondary structure formation. Furthermore, using MbpAgo to cleave RNA is more convenient and cost-effective, for the synthesis of $5^{\prime} \mathrm{OH}$ DNA guides is easier and more inexpensive than 5'P DNA guides. Thus, MbpAgo can potentially be applied in RNA-centric in vivo and in vitro methods such as nucleic acid detection, RNA targeting, and antiviral $(30,39)$. In conclusion, we have demonstrated that MbpAgo is a unique programmable nuclease that has a strong preference for RNA targets and can potentially be widely used in the field of nucleic acid biotechnology.

\section{REFERENCES}

1. Swarts,D.C., Makarova,K., Wang,Y., Nakanishi,K., Ketting,R.F., Koonin,E.V., Patel,D.J. and van der Oost,J. (2014) The evolutionary journey of Argonaute proteins. Nat. Struct. Mol. Biol., 21, 743-753.

2. Koonin,E.V. (2017) Evolution of RNA- and DNA-guided antivirus defense systems in prokaryotes and eukaryotes: common ancestry vs convergence. Biol. Direct, $12,5$.

3. Swarts,D.C., Jore,M.M., Westra,E.R., Zhu,Y., Janssen,J.H., Snijders,A.P., Wang,Y., Patel,D.J., Berenguer,J., Brouns,S.J.J.J. et al. (2014) DNA-guided DNA interference by a prokaryotic Argonaute. Nature, 507, 258-261. 
4. Swarts,D.C., Hegge,J.W., Hinojo,I., Shiimori,M., Ellis,M.A., Dumrongkulraksa,J., Terns,R.M., Terns,M.P. and van der Oost,J. (2015) Argonaute of the archaeon Pyrococcus furiosus is a DNA-guided nuclease that targets cognate DNA. Nucleic Acids Res., 43, 5120-5129.

5. Lisitskaya,L., Aravin,A.A. and Kulbachinskiy,A. (2018) RNA interference and beyond: structure and functions of prokaryotic Argonaute proteins. Nat. Commun., 9, 5165.

6. Kuzmenko,A., Oguienko,A., Esyunina,D., Yudin,D., Petrova,M., Kudinova,A., Maslova,O., Ninova,M., Ryazansky,S., Leach,D. et al. (2020) DNA targeting and interference by a bacterial Argonaute nuclease. Nature, 10.1038/s41586-020-2605-1. Advance online publication.

7. Jolly,S.M., Gainetdinov,I., Jouravleva,K., Zhang,H., Strittmatter,L., Bailey,S.M., Hendricks,G.M., Dhabaria,A., Ueberheide,B. and Zamore,P.D. (2020) Thermus thermophilus Argonaute functions in the completion of DNA replication. Cell, 182, 1545-1559.e18.

8. Ryazansky,S., Kulbachinskiy,A. and Aravin,A.A. (2018) The expanded universe of prokaryotic Argonaute proteins. mBio, 9, e01935-18.

9. Hegge,J.W., Swarts,D.C. and van der Oost,J. (2017) Prokaryotic Argonaute proteins: novel genome-editing tools? Nat. Rev. Microbiol., 16, 5-11.

10. Elkayam,E., Kuhn,C.D., Tocilj,A., Haase,A.D., Greene,E.M., Hannon,G.J. and Joshua-Tor,L. (2012) The structure of human argonaute-2 in complex with miR-20a. Cell, 150, 100-110.

11. Sheng,G., Zhao,H., Wang,J., Rao,Y., Tian,W., Swarts,D.C., van der Oost,J., Patel,D.J. and Wang,Y. (2014) Structure-based cleavage mechanism of Thermus thermophilus Argonaute DNA guide strand-mediated DNA target cleavage. PNAS, 111, 652-657.

12. Nakanishi,K., Weinberg,D.E., Bartel,D.P. and Patel,D.J. (2012) Structure of yeast Argonaute with guide RNA. Nature, 486, 368-374.

13. Kaya,E., Doxzen,K.W., Knoll,K.R., Wilson,R.C., Strutt,S.C., Kranzusch,P.J. and Doudna,J.A. (2016) A bacterial Argonaute with noncanonical guide RNA specificity. PNAS, 113, 4057-4062.

14. Wu,J., Yang,J., Cho,W. C. and Zheng,Y. (2020) Argonaute proteins: Structural features, functions and emerging roles. J. Adv. Res., 24, 317-324.

15. Zander,A., Willkomm,S., Ofer,S., van Wolferen,M., Egert,L., Buchmeier,S., Stockl,S., Tinnefeld,P., Schneider,S., Klingl,A. et al. (2017) Guide-independent DNA cleavage by archaeal Argonaute from Methanocaldococcus jannaschii. Nat. Microbiol., 2, 17034.

16. Willkomm,S., Oellig,C.A., Zander,A., Restle,T., Keegan,R., Grohmann,D. and Schneider,S. (2017) Structural and mechanistic insights into an archaeal DNA-guided Argonaute protein. Nat. Microbiol., 2, 17035.

17. Kuzmenko,A., Yudin,D., Ryazansky,S., Kulbachinskiy,A. and Aravin,A.A. (2019) Programmable DNA cleavage by Ago nucleases from mesophilic bacteria Clostridium butyricum and Limnothrix rosea. Nucleic Acids Res., 47, 5822-5836.

18. Hegge,J.W., Swarts,D.C., Chandradoss,S.D., Cui,T.J., Kneppers,J., Jinek,M., Joo,C. and van der Oost,J. (2019) DNA-guided DNA cleavage at moderate temperatures by Clostridium butyricum Argonaute. Nucleic Acids Res, 47, 5809-5821. 
19. Olina,A., Kuzmenko,A., Ninova,M., Aravin,A.A., Kulbachinskiy,A. and Esyunina,D. (2020) Genome-wide DNA sampling by Ago nuclease from the cyanobacterium Synechococcus elongatus. RNA Biol., 17, 677-688.

20. Liu,Y., Li,W., Jiang,X., Wang,Y., Zhang,Z., Liu,Q., He,R., Chen,Q., Yang,J., Wang,L. et al. (2021) A programmable omnipotent argonaute nuclease from mesophilic bacteria Kurthia massiliensis. Nucleic Acids Res., 49, 1597-1608.

21. Kropocheva,E., Kuzmenko,A., Aravin,A.A., Esyunina,D. and Kulbachinskiy,A. (2021) A programmable pAgo nuclease with universal guide and target specificity from the mesophilic bacterium Kurthia massiliensis. Nucleic Acids Res., 49, 4054-4065.

22. Cao,Y., Sun,W., Wang,J., Sheng,G., Xiang,G., Zhang,T., Shi,W., Li,C., Wang,Y., Zhao,F. et al. (2019) Argonaute proteins from human gastrointestinal bacteria catalyze DNA-guided cleavage of single- and double-stranded DNA at $37^{\circ} \mathrm{C}$. Cell Discov., 5, 38.

23. Swarts,D.C., Szczepaniak,M., Sheng,G., Chandradoss,S.D., Zhu,Y., Timmers,E.M., Zhang,Y., Zhao,H., Lou,J., Wang,Y. et al. (2017) Autonomous generation and loading of DNA guides by bacterial Argonaute. Mol. Cell, 65, 985-998.

24. Enghiad,B. and Zhao,H. (2017) Programmable DNA-guided artificial restriction enzymes. ACS Synth. Biol., 6, 752-757.

25. Song,J., Hegge,J.W., Mauk,M.G., Chen,J., Till,J.E., Bhagwat,N., Azink,L.T., Peng,J., Sen,M., Mays,J. et al. (2020) Highly specific enrichment of rare nucleic acid fractions using Thermus thermophilus argonaute with applications in cancer diagnostics. Nucleic Acids Res., 48, e19.

26. He,R., Wang,L., Wang,F., Li,W., Liu,Y., Li,A., Wang,Y., Mao,W., Zhai,C. and Ma,L. (2019) Pyrococcus furiosus Argonaute-mediated nucleic acid detection. Chem. Commun. (Camb)., $55,13219-13222$

27. Wang,F., Yang,J., He,R., Yu,X., Chen,S., Liu,Y., Wang,L., Li,A., Liu,L., Zhai,C. et al. (2021) PfAgo-based detection of SARS-CoV-2. Biosens. Bioelectron., 177, 112932.

28. Wang,L., He,R., Lv,B., Yu,X., Liu,Y., Yang,J., Li,W., Wang,Y., Zhang,H., Yan,G. et al. (2021) Pyrococcus furiosus Argonaute coupled with modified ligase chain reaction for detection of SARS-CoV-2 and HPV. Talanta, 227, 122154

29. Wang,Y., Juranek,S., Li,H., Sheng,G., Tuschl,T. and Patel,D.J. (2008) Structure of an argonaute silencing complex with a seed-containing guide DNA and target RNA duplex. Nature, 456, 921-926.

30. Abbott,T.R., Dhamdhere,G., Liu,Y., Lin,X., Goudy,L., Zeng,L., Chemparathy,A., Chmura,S., Heaton,N.S., Debs, R. et al. (2020) Development of CRISPR as an Antiviral Strategy to Combat SARS-CoV-2 and Influenza. Cell, 181(4), 865-876.e12.

31. Zadeh,J.N., Steenberg,C.D., Bois,J.S., Wolfe,B.R., Pierce,M.B., Khan,A.R., Dirks,R.M. and Pierce,N.A. (2011) NUPACK: Analysis and design of nucleic acid systems. J. Comput. Chem., 32(1), 170-173.

32. Ye,S., Bae,T., Kim,K., Habib,O., Lee,S.H., Kim,Y.Y, Lee,K.-I., Kim,S. and Kim,J.-S. (2017) DNA-dependent RNA cleavage by the Natronobacterium gregoryi. biorXiv, 10.1101/101923, 20-January-2017, preprint: not peer reviewed. 
33. Pankratov,T.A., Tindall,B.J., Liesack,W. and Dedysh,S.N. (2007) Mucilaginibacter paludis gen. nov., sp. nov. and Mucilaginibacter gracilis sp. nov., pectin-, xylan- and laminarin-degrading members of the family Sphingobacteriaceae from acidic Sphagnum peat bog. Int. J. Syst. Evol. Microbiol., 57(Pt 10), 2349-2354.

34. Willkomm,S., Zander,A., Grohmann,D. and Restle,T. (2016) Mechanistic insights into archaeal and human argonaute substrate binding and cleavage properties. PLoS One, 11, e0164695.

35. Liu,Y., Esyunina,D., Olovnikov,I., Teplova,M., Kulbachinskiy,A., Aravin,A.A. and Patel,D.J. (2018) Accommodation of helical imperfections in Rhodobacter sphaeroides Argonaute ternary complexes with guide RNA and target DNA. Cell Rep., 24, 453-462.

36. Lima,W.F., Wu,H., Nichols,J.G., Sun,H., Murray,H.M. and Crooke,S.T. (2009) Binding and cleavage specificities of human Argonaute2. J. Biol. Chem., 284, 26017-26028.

37. Sheng,G., Zhao,H., Wang,J., Rao,Y., Tian,W., Swarts,D.C., van der Oost,J., Patel,D.J. and Wang,Y. (2014) Structure-based cleavage mechanism of Thermus thermophilus Argonaute DNA guide strand-mediated DNA target cleavage. PNAS, 111, 652-657.

38. Dayeh,D.M., Cantara,W.A., Kitzrow,J.P., Musier-Forsyth,K. and Nakanishi,K. (2018) Argonaute-based programmable RNase as a tool for cleavage of highly-structured RNA. Nucleic Acids Res, 46, e98.

39. Abudayyeh,O.O., Gootenberg,J.S., Essletzbichler,P., Han,S., Joung,J., Belanto,J.J., Verdine,V., Cox,D., Kellner,M.J., Regev,A. et al. (2017) RNA targeting with CRISPR-Cas13. Nature, 550(7675), 280-284. 
TABLE AND FIGURES LEGENDS

A

B

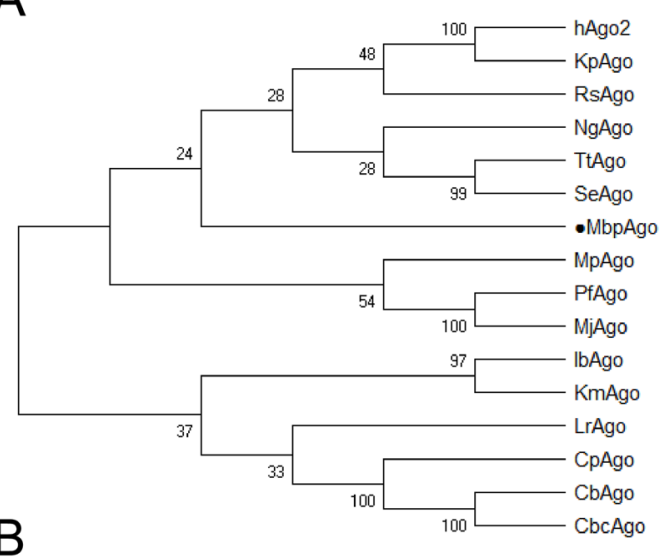

gDNA 5'OH/P-TGAGGTAGTAGGTTGTAT-3'

tRNA

tDNA

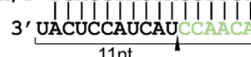

3, TACTCCATCAT

gRNA 5'OH/P-UGAGGUAGUAGGUUGTAU-3'

$\begin{array}{lll}\text { M1 } & \mathbf{3}^{\prime} \text { CCAACAUAUCAUUCGAACCGUGACCGGCAGCAAA-FAM } & \mathbf{5}^{\prime} \\ \text { M2 } & \mathbf{3}^{\prime} \text { CCAACATATCATTCGAACCGTGACCGGCAGCAAA-FAM } & \mathbf{5}^{\prime}\end{array}$
C

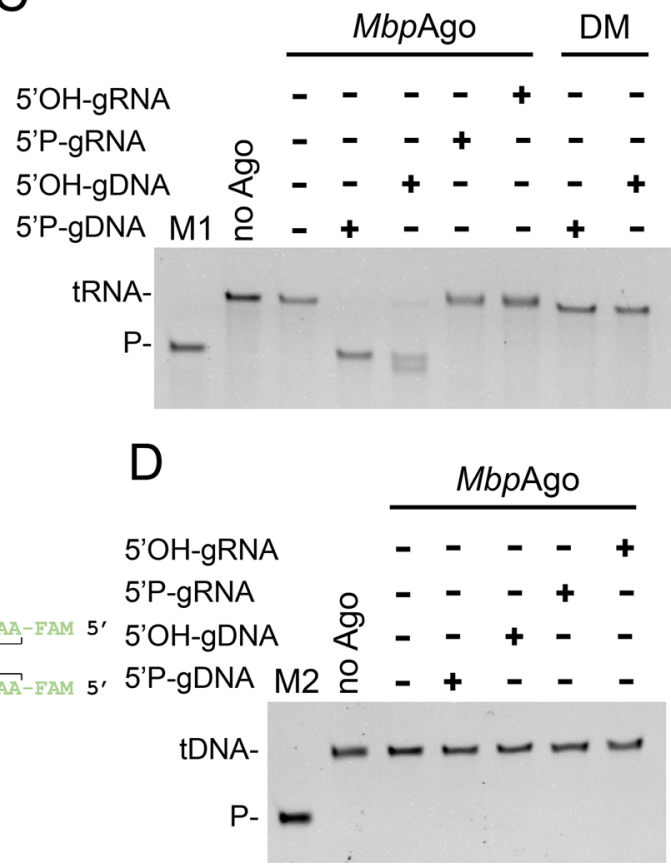

Figure 1. MbpAgo exhibits DNA-guided RNA endonuclease activity at $37^{\circ} \mathrm{C}$. (A) Maximum likelihood phylogenetic tree of characterized Ago proteins. (B) Guide and target oligonucleotides. DNA guides and RNA targets were used in most experiments. Black triangle indicates the cleavage site. (C) MbpAgo exhibits DNA-guided RNA endonuclease activity. (D) MbpAgo exhibits no DNA cleavage activity. Positions of the cleavage products $(P)$ are indicated on the left of the gels. MbpAgo, guides and targets were mixed in a 4:2:1 molar ratio ( $800 \mathrm{nM}$ MbpAgo preloaded with $400 \mathrm{nM}$ guide, plus $200 \mathrm{nM}$ target) and incubated for $30 \mathrm{~min}$ at $37^{\circ} \mathrm{C}$. Catalytic mutant MbpAgo_DM (DM) was used as a control. Lanes M1 and M2 contain chemically synthesized 34-nt RNA and DNA corresponding to the cleavage products of target RNA and target DNA, respectively. 

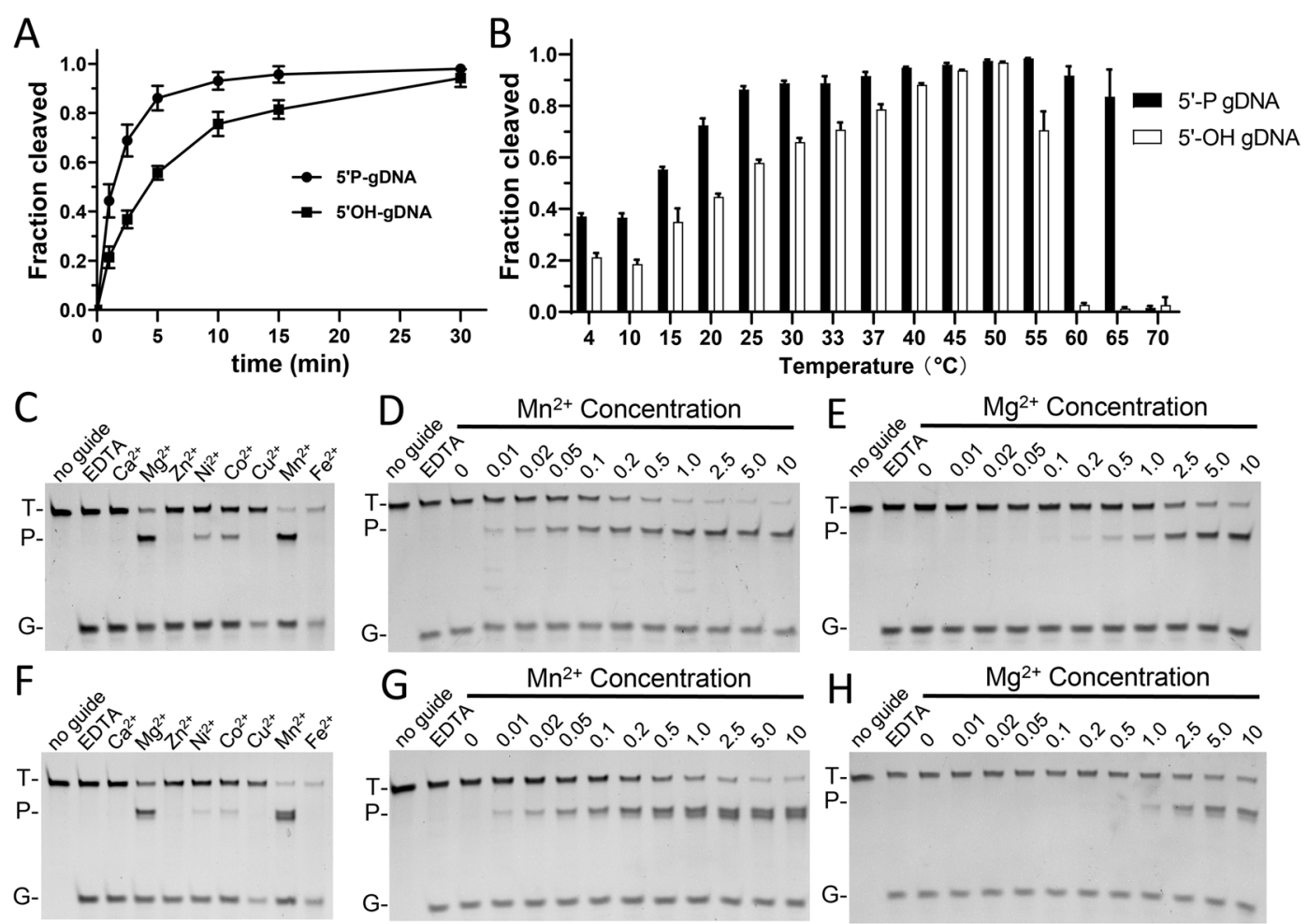

Figure 2. MbpAgo can utilize both 5'-phosphorylated and 5'-hydroxylated DNA guides to cleave RNA targets at $37^{\circ} \mathrm{C}$. (A) Kinetics of nucleic acid cleavage by MbpAgo measured with FAM-labeled RNA targets. (B) Temperature dependence of RNA cleavage by MbpAgo. The assay was performed for 15 min at indicated temperatures. (C) 5'-phosphorylated DNA-guided RNA cleavage by MbpAgo with various divalent cation. (D) and (E) Effects of $\mathrm{Mn}^{2+}$ concentration or $\mathrm{Mg}^{2+}$ concentration on RNA cleavage activity mediated by 5'-phosphorylated DNA guides. (F) 5'-hydroxylated DNA-guided RNA cleavage by MbpAgo with various divalent cation. $(\mathrm{G})$ and $(\mathrm{H})$ Effects of $\mathrm{Mn}^{2+}$ concentration or $\mathrm{Mg}^{2+}$ concentration on RNA cleavage activity mediated by 5'-hydroxylated DNA guides. Positions of the guides $(G)$, targets $(T)$ and cleavage products $(P)$ are indicated on the left of the gels. Experiments in (C) (D) (E) $(F)(G)(H)$ were carried out for $30 \mathrm{~min}$ at $37^{\circ} \mathrm{C}$. 


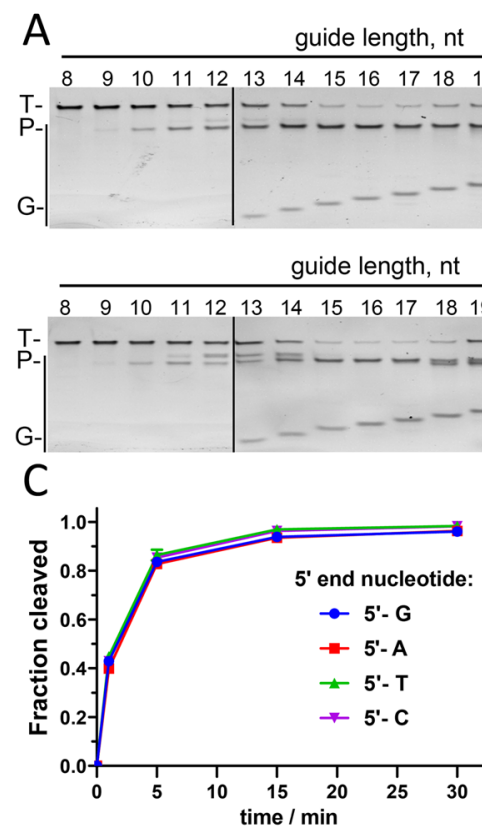

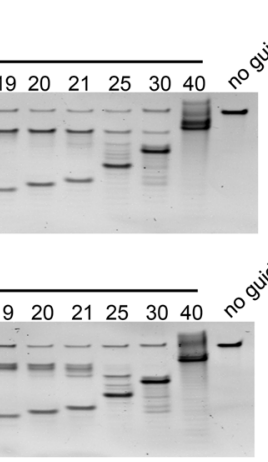

D

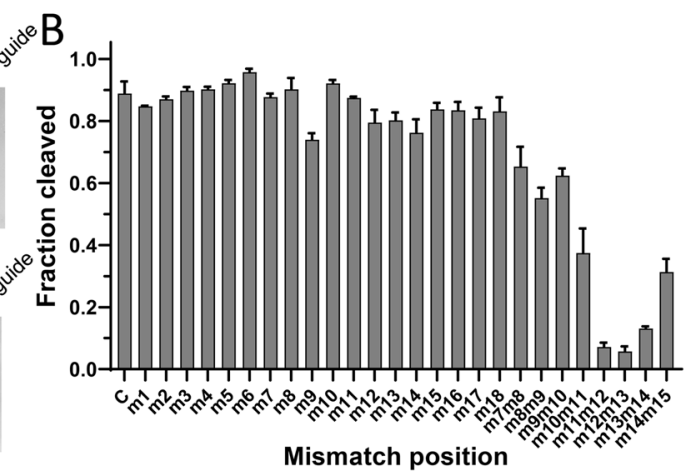

$37^{\circ} \mathrm{C}$

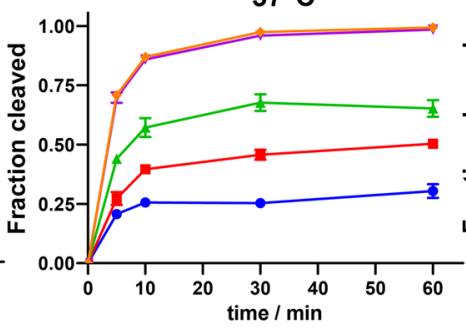

Mismatch position

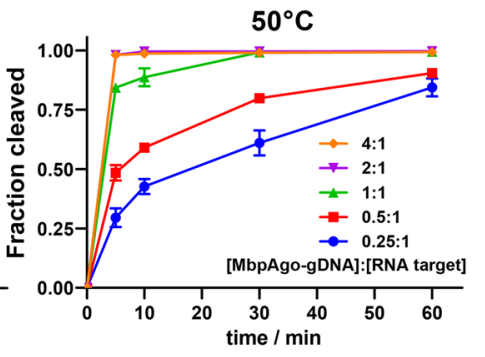

Figure 3. Effects of the guide on RNA cleavage by MbpAgo. (A) Cleavage assay with 5'phosphorylated (Upper panel) and 5'-hydroxylated DNA guides (Lower panel) of varied length. (B) Effects of guide-target mismatches on RNA cleavage by MbpAgo. (C) Preferences for the 5'-guide nucleotides. (D) Quantified data of the MbpAgo-mediated DNA-guided RNA cleavage turnover experiments using 2 pmol RNA target and increasing concentrations of MbpAgo-gDNA (0.5-8 pmol) at $37^{\circ} \mathrm{C}$ (left panel) and $50^{\circ} \mathrm{C}$ (right panel). Experiments in (B) (C) (D) were carried out with $5^{\prime}$ phosphorylated DNA guide. 


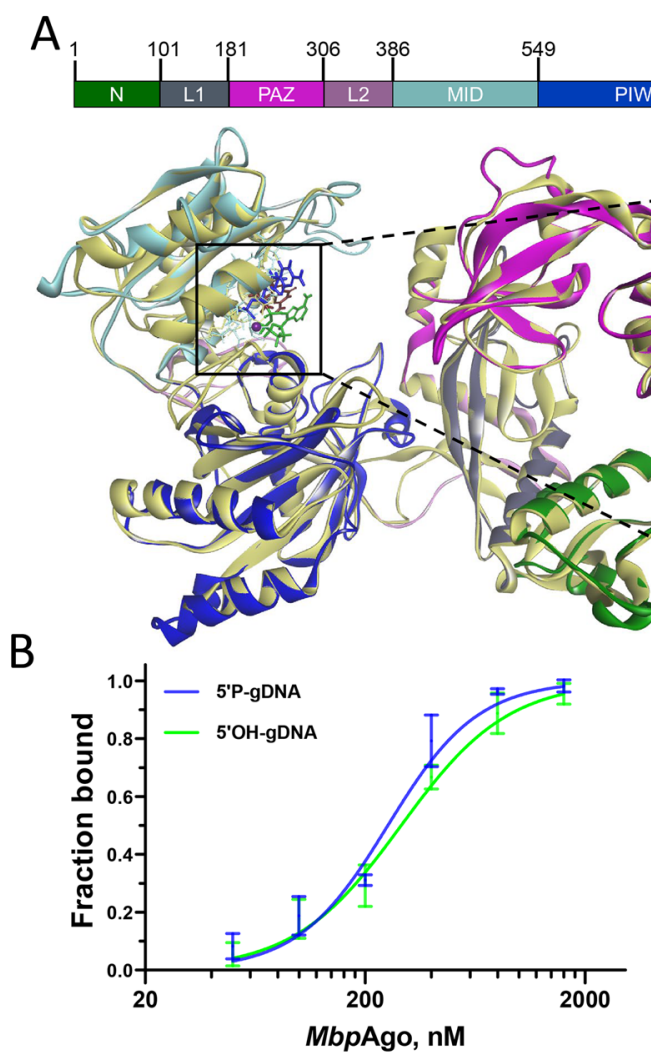

CbAgo: YKQTNK MbpAgo: HKQNYE

Figure 4. Binding analysis of guides and targets by MbpAgo. (A) (left panel) A 3D model of the MbpAgo aligned to the structure of CbAgo in complex with gDNA and tDNA (with bound $\mathrm{Mg}^{2+}$ ion; PDB: 6QZK). Domains of MbpAgo are coloured according to the coloured domain architecture of MbpAgo with residues numbered and CbAgo is coloured with light yellow. The model was built using the SWISS-MODEL portal. (right panel) Amino acid residues of the conserved MID-domain motif (shown for MbpAgo and CbAgo above the structure) and $\mathrm{Mg}^{2+}$ ion (purple) involved in interactions with the first nucleotide (blue) and the second nucleotide (deep red) of guide are highlighted.

Elements of the secondary structure and amino residues specific to MbpAgo and CbAgo are shown in cyan and light yellow, respectively. (B) Binding of $18 \mathrm{nt}$ guide by MbpAgo. The fraction of bound guide was plotted against protein concentration and fitted using the model of specific binding with the Hill slope. MpAgo binds the 5'P-gDNA and 5'OH-gDNA with average $\mathrm{Kd}$ values of $253.7 \pm 23.3 \mathrm{nM}$ and $291.8 \pm 25.2 \mathrm{nM}$, respectively. Data are represented as the mean \pm SD from three independent experiments. (C) Binding of guide: target duplex by MbpAgo. The fraction of bound guide: target duplex was plotted against protein concentration and fitted using the model of specific binding with the Hill slope. MbpAgo binds the 5'P-gDNA: tRNA duplex and 5'OH-gDNA: tDNA duplex with average Kd values of $203.9 \pm 27.2 \mathrm{nM}$ and $269.2 \pm 36.5 \mathrm{nM}$, respectively. Data are represented as the mean $\pm \mathrm{SD}$ from three independent experiments. 
A

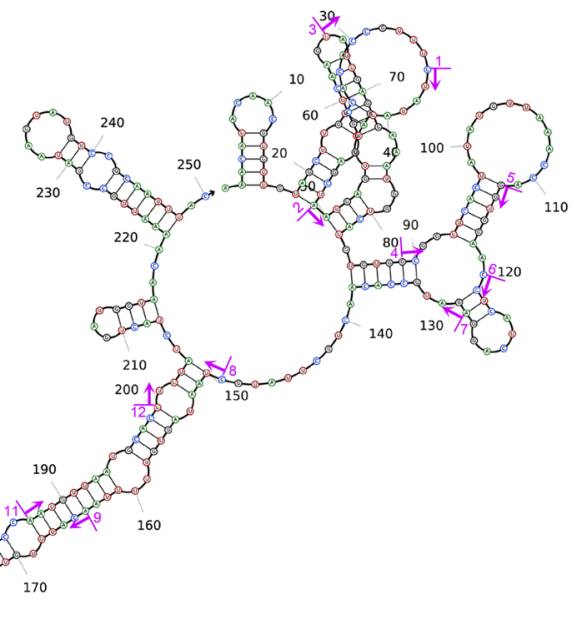

C

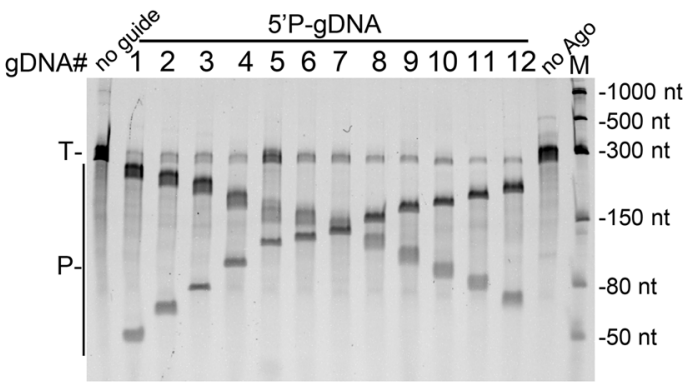

B

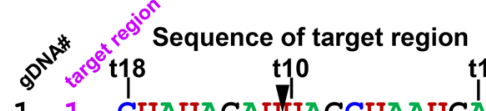

11 CUAUAGAUUAGCUAAUGA

22 AAUGAGUG'UGCUCAAGUA

$3 \quad 3$ UAUUGAGU'GAAAUGGUCA

$4 \quad 4$ GCGGUUCAICUAUAUGUUA

$5 \quad 5$ GGUGGAACICUCAUCAGGA

$6 \quad 6 \quad$ CCUCAUCA'GGAGAUGCCA

$7 \quad 7$ AGAUGCCAICAACUGCUUA

88 CUAAUAGUIGUUUUUAACA

99 ACAUUUGUICAAGCUGUCA

1010 CUGUCACG'GCCAAUGUUA

1111 AAUGUUAATGCACUUUUA

1212 UUUUAUCUACUGAUGGUA IIIII I I I I I II

D

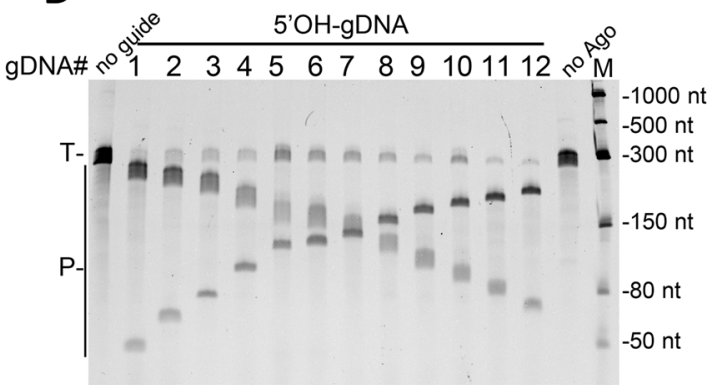

Figure 5. Cleavage of highly structured SARS-CoV-2 RdRp partial RNA by MbpAgo-gDNA complex.

(A) Secondary structure of the SARS-CoV-2 RdRp partial RNA used in the experiments predicted by NUPACK. DNA guides targeting the different target regions of the SARS-CoV-2 RdRp RNA are indicated by purple bars and arrows (1-12). (B) Schematic overview of the designed target regions in the SARS-CoV-2 RdRp RNA. Dotted line flanked by black arrowheads indicates cleavage site. (C) Analysis of the cleavage products obtained after incubation of 5'P-gDNA-MbpAgo complex with SARS-CoV-2 RdRp RNA. (D) Analysis of the cleavage products obtained after incubation of 5 ' $\mathrm{OH}-$ gDNA-MbpAgo complex with SARS-CoV-2 RdRp RNA. Experiments in (C) and (D) were carried out with $5 \mathrm{mM} \mathrm{Mn}^{2+}$. 